\title{
Internacionalização da produção científica brasileira: políticas, estratégias e medidas de avaliação
}

\section{Internationalization of Brazilian scientific output: policies, strategies and assessment measures}

\section{Internacionalización de la producción científica brasileña: políticas, estrategias y medidas de evaluación}

http://dx.doi.org/10.21713/2358-2332.2016.v13.923

Dirce Maria Santin, doutoranda em Comunicação e Informação na Universidade Federal do Rio Grande do Sul (UFRGS) e bibliotecária do Instituto de Ciências Básicas da Saúde da UFRGS, Porto Alegre, RS, Brasil. E-mail: dirce.santin@ufrgs.br.

Samile Andrea de Souza Vanz, doutora em Comunicação e Informação pela Universidade Federal do Rio Grande do Sul (UFRGS) e professora adjunta do Departamento de Ciências da Informação e do Programa de Pós-Graduação em Comunicação e Informação da UFRGS, Porto Alegre, RS, Brasil. E-mail: samilevanz@terra.com.br.

Ida Regina Chittó Stumpf, doutora em Ciências da Comunicação pela Universidade de São Paulo (USP) e professora titular aposentada do Departamento de Ciências da Informação da Universidade Federal do Rio Grande do Sul (UFRGS), Porto Alegre, RS, Brasil. E-mail: irstumpf@ufrgs.br.

\section{Resumo}

A internacionalização da produção científica tem reflexos significativos na ciência contemporânea e na inserção internacional dos países em desenvolvimento. Apesar dos esforços empreendidos pelo Brasil nas últimas décadas, a produção científica nacional ainda enfrenta entraves em relação ao alcance e ao impacto internacional das publicações. Este artigo aborda as políticas e as estratégias voltadas 
à internacionalização da produção científica brasileira e apresenta algumas medidas de avaliação. A internacionalização da produção é discutida com base nos aspectos difusão, colaboração e impacto internacional, com vistas a refletir sobre as possibilidades de ampliar a projeção da ciência brasileira no meio científico internacional.

Palavras-chave: Produção Científica. Internacionalização. Difusão. Colaboração Internacional. Impacto Internacional.

\section{Abstract}

The internationalization of scientific output has a significant impact on the contemporary science and international integration of the developing countries. Despite efforts by Brazil in recent decades, the national scientific output still faces obstacles in relation to the reach and international impact of publications. This article discusses the policies and strategies for internationalization of Brazilian scientific output and presents some evaluation measures. The internationalization of output is discussed based on the aspects of international diffusion, collaboration and impact, in order to reflect on the possibilities of expanding the projection of Brazilian science in the international scientific community.

Keywords: Scientific Output. Internationalization. Diffusion. International Collaboration. International impact.

\section{Resumen}

La internacionalización de la producción científica tiene reflejos significativos en la ciencia contemporánea y en la inserción internacional de los países en desarrollo. A pesar de los esfuerzos de Brasil en las últimas décadas, la producción científica nacional aún enfrenta obstáculos con relación al alcance e impacto internacional de las publicaciones. Este artículo aborda las políticas y estrategias para la internacionalización de la producción científica brasileña y presenta algunas medidas de 
evaluación. La internacionalización de la producción se analiza con base en los aspectos de difusión, colaboración e impacto internacional, con el fin de reflexionar sobre las posibilidades de ampliar la proyección de la ciencia brasileña en la comunidad científica internacional.

Palabras clave: Producción Científica. Internacionalización. Difusión. Colaboración Internacional. Impacto Internacional.

\section{INTRODUÇÃO}

A produção científica brasileira registrou altos índices de crescimento nas últimas décadas, colocando o Brasil na $13^{a}$ posição do ranking mundial de produção científica no início do século XXI. Em 2014, o país registrou um volume de 507.424 artigos de circulação nacional (publicados em periódicos brasileiros) e 576.651 artigos de circulação internacional (veiculados em periódicos estrangeiros), o que corresponde a $2,7 \%$ dos artigos publicados no mundo. Apesar do crescimento, viabilizado em parte pelos investimentos públicos na qualificação de recursos humanos para a ciência e pela ampliação da presença dos periódicos brasileiros em bases de dados internacionais, a ciência brasileira ainda enfrenta entraves em relação ao alcance internacional e ao impacto obtido pelas publicações (THOMSON REUTERS, 2011; PACKER, 2011; LETA, 2012; CRUZ, 2013, CNPQ, 2015).

A internacionalização da produção científica é uma das principais preocupações da comunidade científica dos países emergentes na atualidade. O alcance internacional da produção tem constituído questão central nos debates sobre os rumos da ciência no século $X X I$, e as políticas e estratégias de internacionalização são cada vez mais frequentes no mundo todo. No Brasil, a ampliação dos acordos de cooperação em Ciência, Tecnologia e Inovação (CT\&I) firmados com instituições estrangeiras tem contribuído para superar restrições da posição periférica do país em relação à chamada "zona central” da ciência (SCHOTT, 1998; RUSSELL, 2000). Esses acordos fortalecem a formação de recursos humanos para a pesquisa e oportunizam o intercâmbio de 
professores, pesquisadores e estudantes. Entretanto, as estratégias para o fomento da colaboração internacional ainda são recentes, e diversas ações de internacionalização se sustentam em iniciativas das associações profissionais e dos próprios pesquisadores ou grupos de pesquisa.

A colaboração internacional é uma das expressões mais explícitas da internacionalização da CT\&l, pois revela a associação dos países em projetos de pesquisa colaborativos e integra recursos e competências para o alcance de objetivos comuns. Entretanto, a internacionalização tem caráter mais amplo e envolve outras dimensões além da colaboração (RICYT, 2007; SEBASTIÁN, 2008). Aspectos como a difusão dos resultados das pesquisas em periódicos internacionais, as citações recebidas de autores estrangeiros e a ampliação do enfoque internacional dos periódicos brasileiros também podem contribuir para a internacionalização da produção científica brasileira.

A internacionalização não é consenso nas diversas áreas, e as diferenças entre as disciplinas precisam ser consideradas. O olhar sobre os processos de internacionalização da produção científica deve levar em conta a diversidade e a heterogeneidade das diversas áreas, além do contexto econômico, político, sociocultural e geográfico em que ocorrem as atividades científicas. Com base em tal perspectiva, este artigo apresenta uma discussão sobre a internacionalização da ciência e revisa trabalhos que propõem formas de avaliá-la. Três dimensões de internacionalização são apresentadas - difusão, colaboração e impacto -, a fim de refletir sobre as possibilidades de ampliar a projeção internacional da ciência brasileira. Embora a internacionalização não espelhe a realidade e o interesse das diversas áreas, configura-se como importante fenômeno que permeia a pesquisa brasileira desde o final do século XX. Acredita-se, portanto, que a reflexão sobre a questão é fundamental, bem como o debate sobre as possíveis estratégias de integração da produção científica brasileira com o conhecimento científico mundial. 


\section{INTERNACIONALIZACÃO DA CIÊNCIA}

A trajetória da ciência moderna revela que as principais descobertas científicas estiveram frequentemente ligadas à perspectiva internacional. A internacionalização da ciência pode ser associada a diversos fatores, entre os quais se destacam a crescente complexidade e a interdisciplinaridade das pesquisas; os avanços das tecnologias de informação e comunicação; a facilidade de mobilidade global; as políticas e os instrumentos de cooperação internacional; e a ampliação da colaboração entre autores e instituições do mundo todo (SEBASTIÁN, 2008).

O processo de internacionalização está condicionado às especificidades de cada área, país ou instituição, com suas culturas de publicação. O reconhecimento da diversidade e da heterogeneidade de contextos e disciplinas tem grande importância no estabelecimento de políticas e instrumentos de internacionalização em países e instituições (FIORIN, 2007). A colaboração internacional, por exemplo, varia em função das áreas do conhecimento e costuma ocorrer em maior medida nas áreas básicas e de interesse internacional do que nas áreas aplicadas (FRAME; CARPENTER, 1979).

Outro aspecto relevante em relação à comunicação dos resultados das pesquisas é sua amplitude em termos geográficos. Enquanto os resultados das pesquisas de cunho aplicado são geralmente de interesse regional, a pesquisa nas disciplinas básicas tem apelo internacional, pois trata de temas de interesse universal (FRAME; CARPENTER, 1979). Compreende-se, portanto, que a natureza básica ou aplicada das pesquisas pode influenciar a escolha de canais diferenciados para a comunicação dos resultados.

A internacionalização tem merecido especial atenção por parte de pesquisadores e governos dos países em desenvolvimento. Enquanto os trabalhos produzidos em países desenvolvidos têm forte difusão internacional e são considerados de grande valor para a ciência global, as pesquisas realizadas em países periféricos precisam vencer diversos obstáculos, como aspectos linguísticos e barreiras culturais, 
para obter reconhecimento internacional. Como resultado, as estratégias de internacionalização desses países se baseiam principalmente na colaboração com autores de países desenvolvidos, buscando ampliar o alcance e a visibilidade de suas pesquisas (RUSSELL, 2000).

No Brasil, as perspectivas de inserção da pesquisa no meio científico internacional têm se alterado significativamente nos últimos anos. Essa tendência reflete o resultado dos esforços realizados em longo prazo para sistematizar e internacionalizar a atividade científica do país, com participação intensa dos programas de pós-graduação. A pós-graduação brasileira, por exemplo, adota políticas e estratégias de internacionalização há algumas décadas. As ações de internacionalização acadêmica, desenvolvidas com o apoio das agências de fomento nacionais, como a Coordenação de Aperfeiçoamento de Pessoal de Nivel Superior (Capes) e o Conselho Nacional de Desenvolvimento Científico e Tecnológico (CNPq), têm resultado no intercâmbio de estudantes, de pesquisadores e de experiências não apenas no percurso que vai do Brasil para outros países, mas também na vinda de estudantes e de pesquisadores estrangeiros para atuar no país (MARRARA, 2007).

A internacionalização relativa à colaboração e à difusão da produção científica também tem sido um dos critérios adotados pela Capes para a avaliação dos programas de pós-graduação. A inserção internacional da ciência brasileira foi um dos eixos da Estratégia Nacional de Ciência, Tecnologia e Inovação 2012-2015. O documento destacava a Ciência, Tecnologia e Inovação (CT\&I) como elemento decisivo nas parcerias estratégicas internacionais, especialmente em relação aos grupos formados pelos BRICS (Brasil, Rússia, Índia, China e África do Sul) e IBAS (Índia, Brasil e África do Sul). As políticas e ações governamentais previam o fomento à internacionalização da ciência e o fortalecimento da cooperação científica e tecnológica com vistas à consolidação de um novo padrão de inserção internacional do país (BRASIL, 2012). A Estratégia 2016-2019 encontra-se em fase de discussão e prevê a definição de temas estratégicos de pesquisa que elevem a competitividade e a inserção internacional do país, além de destacar aspectos como a formação de recursos humanos de nivel internacional; a continuidade dos programas de intercâmbio e o fortalecimento da colaboração internacional (BRASIL, 2015). 
A importância das dimensões internacionais nos processos de geração, difusão e uso do conhecimento científico tem exigido a criação de novos indicadores para medir a evolução, as características e a intensidade da internacionalização da ciência em países e instituições.

O Manual de Indicadores de Internacionalización de la Ciencia y la Tecnología - Manual de Santiago, publicado pela Red Iberoamericana de Indicadores de Ciencia y Tecnologia (RICyT) em 2007, é um dos principais instrumentos de avaliação da internacionalização das atividades de CT\&l. O Manual propõe um conjunto de indicadores para medir a intensidade e as características de internacionalização, tanto no âmbito nacional como no de instituições e organismos internacionais. Os indicadores são classificados de acordo com os componentes dos sistemas de CT\&l, envolvendo políticas e instrumentos de internacionalização; atividades de ciência e tecnologia; e resultados e produtos das atividades de ciência e tecnologia. $O$ último grupo inclui os indicadores de internacionalização da produção científica, classificados em três dimensões principais:

a) difusão internacional-publicação em periódicos internacionais;

b) coautoria - participação de autores de diferentes países nas publicações;

c) impacto internacional - citação recebida de outras publicações internacionais.

O Manual de Santiago representa um esforço de avaliação da internacionalização a partir de uma perspectiva ibero-americana. Abordagem semelhante foi utilizada por Bordons e colaboradores (2006) para analisar a internacionalização da produção espanhola nas áreas de Radiologia e Neuroimagem. Artigo de Luna-Morales (2012) dedicado à produção científica mexicana também examinou a internacionalização com base na difusão internacional, na colaboração com países e instituições estrangeiras e no impacto internacional obtido pelas publicações. No Brasil, estudo de Santin, Vanz e Stumpf (2015) adotou o Manual de Santiago como base para a definição de indicadores de internacionalização da produção científica em Ciências Biológicas de uma instituição do Sul do país e avaliou as dimensões difusão, colaboração e impacto internacional. 
Perspectivas diversas são adotadas em outros estudos. Leite, Mugnaini e Leta (2011) propuseram um indicador para a análise da visibilidade internacional da produção científica. A ideia de internacionalização adotada pelos autores considerou a publicação de artigos em periódicos internacionais, estimada pelo Índice de Publicação Internacional. A análise utilizou os dados dos Currículos Lattes dos pesquisadores brasileiros sobre os artigos publicados entre 1997 e 2004. A proposta se concentra na internacionalização de pesquisadores e de áreas de pesquisa e revela as Ciências Exatas e da Terra como as mais internacionalizadas do país, seguidas pelas Ciências Biológicas.

A internacionalização dos periódicos foi o tema central de estudo de Pinto e Cunha (2008), que concentrou esforços na análise de dois periódicos brasileiros da área de Química. O diferencial do estudo está na análise da internacionalização das publicações não apenas pela indexação em bases de dados internacionais, mas também pela publicação de artigos de autores estrangeiros. Já a preocupação com o impacto das publicações está presente no estudo de Meneghini, Mugnaini e Packer (2006), que avaliaram a orientação nacional e internacional das citações recebidas por periódicos indexados no SciELO e na Web of Science, e no trabalho de Cruz (2013), que apresentou dados sobre as citações nacionais e internacionais recebidas pelos periódicos brasileiros.

Ainda em relação aos periódicos, Buela-Casal e colaboradores (2006) definiram quatro critérios para a análise da internacionalidade: colaboração multinacional; composição do conselho editorial; distribuição multinacional dos editores associados; e distribuição dos autores. 0 estudo também propôs a criação de um índice de internacionalidade baseado na combinação de diversas variáveis, defendendo que critérios únicos não podem ser considerados medidas inequívocas de internacionalidade.

A internacionalização baseada na cooperação com outros países serviu de base para o artigo de Ponds (2009), que analisou a internacionalização da produção científica holandesa. A colaboração internacional também foi adotada por Tan et al. (2015) para propor o 
Índice de Internacionalidade, uma medida de internacionalização dos pesquisadores da Malásia. O artigo avaliou a internacionalização dos pesquisadores em vez de artigos, assemelhando-se, nesse aspecto, ao estudo de Leite, Mugnaini e Leta (2011).

Os indicadores mencionados constituem uma amostra dos modelos utilizados para avaliação da internacionalização da produção científica. Sua validade e adequação devem ser consideradas no contexto de cada disciplina, bem como sua capacidade de servir como insumos para as políticas científicas nacionais e institucionais. Os resultados de análises periódicas baseadas nessas medidas e a criação de novos indicadores voltados à perspectiva internacional são de extrema relevância no planejamento de políticas científicas e no processo de internacionalização das atividades de CT\&l.

Os principais aspectos da internacionalização da produção científica são discutidos a seguir, com base na classificação proposta pela RICyT (2007): difusão, colaboração e impacto internacional.

\subsection{Difusão internacional da produção científica}

Publicar em periódicos internacionais e ampliar o alcance das publicações no espaço de diferentes nações é uma prática consolidada em algumas áreas da ciência brasileira, mas incipiente em diversas outras. A difusão internacional caracteriza-se pela veiculação dos resultados em periódicos internacionais, pela indexação dos periódicos em bases de dados internacionais e pelo uso do idioma inglês nas publicações, entre outros aspectos.

A indexação em bases de dados internacionais constitui característica básica da internacionalização da produção científica (RICYT, 2007). Amplamente discutida na literatura, a indexação também pode ser compreendida como um requisito de visibilidade dos periódicos no cenário internacional. Nessa perspectiva, toda a produção científica brasileira indexada em bases de dados como a Web of Science, a Scopus 
e a PubMed, por exemplo, pode ser considerada internacionalizada no que se refere à difusão internacional.

A difusão internacional também pode ser avaliada pela proporção de artigos publicados em periódicos estrangeiros em relação ao total de artigos de um país ou instituição (RICYT, 2007). Estudos anteriores revelaram a preferência dos pesquisadores brasileiros das Ciências Biológicas e das Ciências Exatas e da Terra pela publicação dos resultados em periódicos estrangeiros (LEITE; MUGNAINI; LETA, 2011; SANTIN; VANZ; STUMPF, 2015). É evidente que não basta publicar fora do Brasil e que é necessário atentar para outras questões, mas a difusão dos artigos em periódicos de outros países favorece a divulgação da produção científica brasileira, especialmente quando veiculada em periódicos de ampla circulação internacional.

A reflexão sobre a internacionalização da produção científica também inclui a questão do idioma como um dos elementos essenciais. Atualmente, o inglês é considerado a língua franca da ciência mundial (MEADOWS, 1999; MENEGHINI; PACKER, 2007; ROYAL SOCIETY, 2011). O uso desse idioma tem influência direta na internacionalização das publicações e reforça a hipótese de que existe uma relação clara entre o uso do idioma inglês na produção científica e sua visibilidade nas instâncias globais. O uso do inglês não é essencial apenas aos artigos publicados em periódicos estrangeiros, mas também aos periódicos brasileiros que pretendem obter alcance internacional.

A internacionalização dos periódicos brasileiros tem se mostrado crescente nas últimas décadas, e as estratégias utilizadas pelos editores envolvem ações como:

a) uso do idioma inglês - veiculação dos artigos em inglês com vistas a ampliar a visibilidade e o impacto das publicações;

b) publicação de artigos de autores estrangeiros - o desafio de atrair autores estrangeiros para submeter manuscritos de qualidade exige mudanças nas políticas editoriais, divulgação de websites e sistemas de gerenciamentos de revistas em inglês; ampliação do quadro de avaliadores; agilidade nos processos editoriais e uma série de esforços para captação e retenção de autores internacionais; 
c) indexação em bases de dados internacionais - característica de consenso em relação à circulação internacional dos periódicos, capaz de conferir visibilidade à produção científica e ampliar seu impacto na comunidade internacional;

d) presença de pesquisadores estrangeiros como editores associados, membros de comitês editoriais e/ou revisores ad hoc - a participação ativa de pesquisadores internacionais pode qualificar as revistas brasileiras e promovê-las junto à comunidade científica internacional;

e) busca de maior impacto - o desafio de ampliar o impacto internacional da ciência brasileira está diretamente ligado ao aumento das citações recebidas por artigos veiculados em periódicos publicados no país. Mesmo os títulos brasileiros indexados na Web of Science costumam receber de três a cinco vezes menos citações por artigo do que os periódicos estrangeiros (CRUZ, 2013). Estratégias editoriais como as apresentadas podem ampliar a visibilidade das publicações e atrair mais citações para os artigos publicados no Brasil.

O Qualis Periódicos - sistema de avaliação de periódicos da Capes e que integra a avaliação do Sistema Nacional de Pós-Graduação no Brasil - estimula a adoção de estratégias de internacionalização pelos periódicos, na medida em que estabelece diversos critérios de avaliação voltados à perspectiva internacional. A Associação Brasileira de Editores Científicos (ABEC) e a Scientific Electronic Library (SciELO) também têm empreendido importantes ações de estímulo à internacionalização dos periódicos e da produção científica brasileira. A essas iniciativas somamse os esforços de órgãos governamentais e de agências de fomento para ampliar o alcance internacional dos periódicos brasileiros e dos artigos neles veiculados.

\subsection{Colaboração internacional}

A colaboração internacional está na base dos processos de internacionalização e tem papel fundamental nas políticas e estratégias de CT\&I. A prática permite reunir experiências de diversos países para 
a resolução de problemas comuns, além de viabilizar o financiamento de equipes e equipamentos. Os artigos resultantes da colaboração internacional são geralmente mais citados e têm, portanto, maior impacto na pesquisa mundial (PERSSON; GLÄNZEL; DANELL, 2004; ADAMS, 2013). Entre as possíveis explicações para o aumento do impacto dessas publicações estão a visibilidade ampliada pela divulgação em periódicos internacionais e a qualidade das publicações, uma vez que é provável que a reunião de grupos de elite para resolução de problemas comuns resulte em pesquisas mais qualificadas.

As motivações para a colaboração internacional são diversas e envolvem tanto os padrões endógenos do campo científico como os benefícios esperados do trabalho conjunto entre as nações. Quatro fatores que favorecem a colaboração foram destacados pela Royal Society (2011) em estudo sobre as redes de colaboração internacional no início do século XXI. São eles:

a) busca por excelência - o que envolve a produção colaborativa com pesquisadores de destaque internacional e permite aos parceiros a ampliação das experiências e da divulgação das publicações. Os laços estabelecidos com pesquisadores de elite favorecem a participação em agendas de pesquisa global e em novas redes de conhecimento. A busca por excelência também se reflete na pesquisa de escala, na qual os projetos são muito grandes para uma única nação e envolvem o compartilhamento de recursos, equipamentos, objetos de pesquisa, entre outros;

b) benefícios da coautoria - que podem ser percebidos especialmente em relação ao impacto alcançado pela produção científica. O estudo da Royal Society (2011) verificou que para cada autor internacional associado a um artigo existe um aumento do número de citações atribuídas ao artigo correspondente, embora este aumento seja menos claro após a contagem de dez autores. O aumento do impacto pode refletir a visibilidade e a qualidade das publicações, enquanto a análise de coautoria e impacto das publicações pode indicar os pares, as instituições e os países prioritários 
para compartilhamento das pesquisas de interesse global e regional;

c) necessidade de colaborar com pesquisadores de outros países, que pode ser mais intensa para os países em desenvolvimento, pois implica ampliar a capacidade da pesquisa pela troca de experiências e pelo acesso a instalações, equipamentos e investimentos muitas vezes limitados nos próprios países. Entretanto, a colaboração também é positiva para os países desenvolvidos, uma vez que o uso de laboratórios altamente equipados pode ser compensado com recursos geográficos únicos, como é o caso de espécies da biodiversidade, por exemplo;

d) potencial geopolítico da colaboração científica, pois a cooperação nas pesquisas também tem reflexos nas relações políticas e diplomáticas entre os países. A política externa pode ver na ciência uma importante ferramenta para reforçar relacionamentos e promover ações de interesse global. A colaboração científica também pode servir como uma ponte entre as nações cujos laços políticos estejam fragilizados por guerras ou conflitos econômicos.

A cooperação com pesquisadores de outros países assume importância estratégica para a internacionalização da ciência, e a produção científica está se tornando cada vez mais colaborativa. Ao traçar um perfil da colaboração internacional de alguns países em mais de três décadas (1981-2012), Adams (2013) constatou a redução do percentual de artigos domésticos em países como Estados Unidos, Reino Unido, Alemanha, França, Holanda e Suíça. Por outro lado, o percentual de artigos domésticos de países como Brasil, China, Índia e Coreia do Sul tem se mantido estável, compreendendo cerca de 75\% de sua produção no período. A Royal Society (2011) avaliou que 35\% dos artigos publicados no mundo na primeira década do século XXI foram produzidos em coautoria internacional, superando o índice de $25 \%$ identificado na década de 1990. No Brasil, a colaboração internacional atinge um índice de aproximadamente 30\%, com redução nos últimos anos e algumas diferenças entre as disciplinas (VANZ; STUMPF, 2012; LETA; THIJS; GLANZEL, 2013). 


\subsection{Impacto internacional}

A comunicação dos resultados das pesquisas potencializa o impacto da ciência nos campos social, cultural, político, econômico e, mais fortemente, no próprio campo científico. Neste último, o impacto se caracteriza pelo uso da informação divulgada em novas pesquisas e demonstra o desempenho da produção científica pelas citações recebidas em estudos posteriores. Alcançar impacto internacional significa integrar as ideias da ciência ao conhecimento global, tornando-o parte da literatura científica pela divulgação dos resultados e pelas citações recebidas em publicações internacionais.

Os indicadores de impacto, representados pelos dados de citações, constituem instrumentos de avaliação do desempenho e da qualidade científica e refletem o prestígio e a influência de autores, instituições e países. A procedência e o vínculo dos autores associados às publicações responsáveis pelas citações também revelam o alcance geográfico da produção. 0 impacto em grande número de países pode indicar a influência e o prestígio de publicações e de autores na comunidade internacional, difundir as ideias da ciência no contexto global e aumentar o potencial de visibilidade dos artigos. As citações recebidas de instituições e grupos de pesquisa estrangeiros também podem revelar similaridade nos temas de pesquisa e oportunizar a análise de possíveis parcerias e acordos de cooperação internacional (VANZ; CAREGNATO, 2003; CRUZ, 2013; ROYAL SOCIETY, 2011).

Alguns fatores que interferem positivamente no impacto internacional da produção científica são apontados a seguir, ainda que o grau de influência possa variar de acordo com os padrões de produção e citação nas diferentes áreas do conhecimento:

a) qualidade das pesquisas e relevância social e econômica trabalhos realizados por equipes altamente qualificadas, abordando temas emergentes e conectados aos problemas globais contemporâneos (como alterações climáticas, água, alimentos, segurança energética e biodiversidade) têm maior apelo internacional e tendem a alcançar maior impacto na ciência global (ROYAL SOCIETY, 2011); 
b) publicação de artigos em periódicos renomados e de ampla circulação internacional - a seleção dos periódicos deve considerar a difusão das publicações e o potencial de visibilidade dos artigos. Elementos como o idioma, o Fator de Impacto e o acesso aberto às publicações podem interferir positivamente no número de citações recebidas pelos artigos;

c) colaboração internacional - a correlação entre o número de autores ou países associados aos artigos e o número de citações recebidas foi confirmada em estudos anteriores, reforçando a hipótese de que a colaboração internacional apresenta reflexos no impacto alcançado pelas publicações (PERSSON; GLÄNZEL; DANELL, 2004; ADAMS, 2013).

O impacto representa um dos principais desafios da ciência brasileira, tendo em vista que o aumento da produtividade de autores brasileiros nas últimas décadas não foi acompanhado por crescimento semelhante no número de citações recebidas pelas publicações. Embora o Brasil seja responsável por 2,7\% da produção mundial, não alcança 0,8\% do impacto relativo ao mundo, ficando abaixo da média de impacto mundial $(1,0 \%)$. As exceções são a área de Física, que atinge um índice de impacto mundial de 1,5\%, e as áreas de Ecologia, Microbiologia, Farmacologia e Toxicologia, que apresentam equilíbrio no ritmo de publicação e citação e mantêm posição especial para a ciência brasileira (CRUZ, 2013; FINK et al., 2014).

\section{CONSIDERACÕES FINAIS}

A ciência moderna teve boas razões para olhar além das fronteiras em busca de parcerias e inspiração científica. Os desafios globais contemporâneos ampliaram a ênfase no alcance internacional da ciência, tornando inter-relacionadas e interdependentes as atividades científicas de regiões e países. Esse cenário provoca mudanças significativas na forma como as nações investem em ciência. Políticas e estratégias são definidas a fim de ampliar o alcance dos resultados e explorar as potencialidades regionais em relação à ciência global. 
A internacionalização da produção científica pode ocorrer de diversas formas e envolve as dimensões difusão, colaboração e impacto internacional. O desafio de internacionalizar a produção científica se revela nos esforços de governos, de organismos internacionais e da própria comunidade científica para a construção de indicadores voltados à perspectiva internacional. Resultados provenientes de metodologias adequadas ganham importância nesse contexto por sua capacidade de apoiar o desenvolvimento e a avaliação de políticas e estratégias de internacionalização da ciência em países e instituições.

Os desafios globais estão no escopo da ciência, mas esta não pode enfrentá-los como elementos únicos. Não basta, portanto, internacionalizar a ciência e a produção científica dela resultante. A pesquisa brasileira ainda precisa enfrentar outros desafios, como a integração com as políticas públicas e a atividade econômica com vistas a gerar benefícios sociais e inovação tecnológica. A atenção aos temas de pesquisa de interesse local também não deve ser diminuída, sob pena de menosprezar potencialidades regionais. A mesma prudência deve ser mantida em relação aos processos de produção e comunicação do conhecimento científico, marcados que são pelas tradições e práticas sociais próprias de cada disciplina.

\section{Referências}

ADAMS, J. Collaborations: the fourth age of research. Nature, London, v. 497, n. 7.451, p. 557-560, 2013.

BUELA-CASAL, G. et al. Measuring internationality: reflections and perspectives on academic journals. Scientometrics, Amsterdam, v. 67, n. 1, p. 45-65, 2006.

BORDONS, M. et al. Internacionalización de la produción científica de España en Radiología y Neuroimagen (1996-2003). Radiología, Madrid, v. 48, n. 3, p. 137-146, 2006

BRASIL. Ministério da Ciência, Tecnologia e Inovação. Estratégia Nacional de Ciência, Tecnologia e Inovação 2012-2015. Brasília, 2012. Disponível 
em: <http://livroaberto.ibict.br/docs/218981.pdf>. Acesso em: 20 fev. 2016.

Proposta da Estratégia Nacional de Ciência, Tecnologia e Inovação 2016-2019. Brasília, 2015. Disponível em: <http://goo. gl/9QMEXm>. Acesso em: 20 fev. 2016.

CONSELHO NACIONAL DE DESENVOLVIMENTO CIENTÍFICO E TECNOLÓGICO - CNPq. Produção CT\&A. Brasília, 2015. Disponível em: <http:/ / lattes.cnpq.br/web/dgp/producao-c-t-a>. Acesso em: 11 dez. 2015.

CRUZ, C. H. B. O desafio de aumentar o impacto da ciência brasileira. São Paulo, 2013. Disponível em:<http:/ /www.fapesp.br/eventos/2013/05/ confap/desafio-impacto-confap.pdf>. Acesso em: 10 jan. 2016.

FINK, D. et al. S\&T knowledge production from 2000 to 2009 in two periphery countries: Brazil and South Korea. Scientometrics, Amsterdam, v. 99, n. 1, p. 37-54, 2014.

FIORIN, J. L. Internacionalização da produção científica: a publicação de trabalhos de Ciências Humanas e Sociais em periódicos internacionais. RBPG, Brasília, v. 4, n. 11, p. 263-281, 2007.

FRAME, J. D.; CARPENTER, M. P. International research collaboration. Social Studies of Science, London, v. 9, n. 4, p. 481-497, 1979.

LEITE, P.; MUGNAINI, R.; LETA, J. A new indicator for international visibility: exploring Brazilian scientific community. Scientometrics, Amsterdam, v. 88, n. 1, p. 311-319, 2011.

LETA, J. Brazilian growth in the mainstream science: the role of human resources and national journals. Journal of Scientometrics Research, New Delhi, v. 1, n. 1, p. 44-52, 2012.

LETA, J.; THIJS, B.; GLÄNZEL, W. A macro-level study of science in Brazil: seven years later. Encontros Bibli, Florianópolis, v. 18, n. 36, p. 51-66, 2013. 
LUNA-MORALES, M. E. La colaboración científica y la internacionalización de la internacionalización de la ciencia mexicana de 1980 a 2004. Investigación Bibliotecológica, México, v. 26, n. 57, p. 103-129, 2012.

MARRARA, T. Internacionalização da pós-graduação: objetivos, formas e avaliação. RBPG, Brasília, v. 4, n. 8, p. 245-262, 2007.

MEADOWS, A. J. A comunicação científica. Brasília: Briquet de Lemos, 1999.

MENEGHINI, R.; MUGNAINI, R.; PACKER, A. L. International versus national oriented Brazilian scientific journals: a scientometric analysis based on SciELO and JCR-ISI databases. Scientometrics, Amsterdam, v. 69, n. 3, p. 529-538, 2006.

MENEGHINI, R.; PACKER, A. L. Is there science beyond English? EMBO Reports, Oxford, v. 8, n. 2, p. 112-116, 2007.

PACKER, A. L. Os periódicos brasileiros e a comunicação da pesquisa nacional. Revista USP, São Paulo, n. 89, p. 26-61, 2011.

PACKER, A. L.; MENEGHINI, R. Visibilidade da produção científica. In: POBLACIÓN, D. A.; WITTER, G. P.; SILVA, J. F. M. Comunicação \& produção científica: contexto, indicadores e avaliação. São Paulo: Angellara, 2006. p. 235-259.

PERSSON, O.; GLÄNZEL, W.; DANELL, R. Inflationary bibliometric values: the role of scientific collaboration and the need for relative indicators in evaluative studies. Scientometrics, Amsterdam, v. 60, n. 3, p. 421-432, 2004.

PINTO, A. C.; CUNHA, A. S. Avaliação da pós-graduação da área de Química na Capes e a internacionalização das revistas da Sociedade Brasileira de Química: Journal of the Brazilian Chemical Society e Química Nova. Química Nova, São Paulo, v. 31, n. 8, p. 221-226, 2008.

PONDS, R. The limits to internationalization of scientific research collaboration. Journal of Technology Transfer, Indianapolis, v. 34, n. 1, p. $76-94,2008$. 
RED IBEROAMERICANA DE INDICADORES EM CIÊNCIA E TECNOLOGIA RICYT. Manual de indicadores de internacionalización de la ciencia y la tecnología (Manual de Santiago). Buenos Aires, 2007.

ROYAL SOCIETY. Knowledge, networks and nations: global scientific collaboration in the 21st century. London: Royal Society, 2011. Disponivel em:<https://royalsociety.org/ /media/Royal_Society_Content/policy/ publications/2011/4294976134.pdf>. Acesso em: 30 nov. 2015.

RUSSELL, J. M. Tecnologias eletrônicas de comunicação: bônus ou ônus para os cientistas dos países em desenvolvimento? In: MULLER, S. P. M.; PASSOS, E. J. L. Comunicação científica. Brasília: Universidade de Brasília, 2000. p. 35-49.

SANTIN, D. M.; VANZ, S. A. S.; STUMPF, I. R. C. Internacionalização da produção científica em Ciências Biológicas da UFRGS: 2000-2011. Transinformação, Campinas, v. 27, n. 3, p. 209-2018, set./dez. 2015.

SCHOTT, T. Ties between center and periphery in the scientific worldsystem: accumulation of rewards, dominance and self-reliance in the center. Journal of World-Systems Research, Baltimore, v. 4, n. 2, p. 112-144, 1998.

SEBASTIÁN, J. El Manual de Santiago: un guía para medir la internacionalização de la I+D. In: ALBORNOZ, M.; VOGT, C.; ALFARAZ, C. (Ed.). Indicadores de ciencia y tecnología em iberoamérica. Buenos Aires: RICYT, 2008. p. 167-193.

TAN, H. X. et al. Impact analysis of domestic and international research collaborations: a Malaysian case study. Scientometrics, Amsterdam, v. 102, n. 1, p. 885-904, 2015.

THOMSON REUTERS. Essential Science Indicators: top 20 countries in all fields. 2012. Disponivel em: <http://archive.sciencewatch.com/dr/ cou/2011/11decALL/>. Acesso em: 15 set. 2015.

VANZ, S. A. S.; CAREGNATO, S. E. Estudos de citação: uma ferramenta para entender a comunicação científica. Em Questão, Porto Alegre, v. 9, n. 2, p. 295-307, 2003. 
VANZ, S. A. S.; STUMPF, I. R. C. Scientific output indicators and scientific collaboration network mapping in Brazil. Collnet Journal, New Delhi, v. 6, p. 1-20, 2012.

Recebido em 12/03/2016 Aprovado em 16/05/2016 